\title{
Deposition of coatings on long-period fiber gratings: tunnel effect analogy
}

\author{
I. Del Villar, I. R. Matias and F. J. Arregui \\ Departamento de Ingeniería Eléctrica y Electrónica, Universidad Pública de Navarra, 31006 \\ Pamplona, Spain.
}

\begin{abstract}
The sensitivity of LPFGs, coated with high-refractive-index thin film overlays, to the refractive index and the thickness of the overlay, and to the ambient refractive index, can be enhanced with a design based on a two-overlay coating of an LPFG. The first overlay of lower refractive index than the cladding affects the guidance of a cladding mode in the second overlay of higher refractive index than the cladding. This causes a more abrupt cladding modal redistribution than with the deposition of a unique high-refractive-index overlay. The phenomenon is analyzed with a method based on a vectorial analysis of modes and the application of coupled mode theory.
\end{abstract}

Keywords: gratings, coupled-mode analysis, thin films, optical fiber sensors. 


\section{Introduction}

Long-period fiber gratings (LPFGs) are becoming increasingly important in the field of telecommunications, especially in the development of sensors devices (James and Tatam 2001). The deposition of a thin overlay on the cladding of an LPFG was reported for the first time by (Rees et al. 2002). The nanodeposition technique used in that case was Langmuir Blodgett (LB). The characteristic attenuation bands in the transmission spectrum experimented important wavelength shifts as a function of the overlay thickness. In addition to this, the depth of the attenuation band could decrease to that point that, for some overlay thickness values, the attenuation bands could vanish. This phenomenon has been corroborated with another nanodeposition technique: Electrostatic-Self Assembly (ESA) method; and the results have been explained (Del Villar et al. $2005 \mathrm{a}, \mathrm{b}$ ) with a theoretical model based on the LP mode approximation (Anemogiannis et al. 2003). On the other hand, dip-coating (DC) technique has been used to prove that a similar phenomenon is observed if an LPFG is coated with a thin overlay and the resonance wavelength shift is analyzed as a function of the ambient refractive index (Pilla et al. 2005 a). In the three nanodeposition techniques the refractive index contrast between the overlay and the cladding was moderate because the materials used for the deposition were polymeric ones. Consequently, the theoretical results fitted the experimental ones. However, if the cladding is coated with a different material of higher refractive index than polymers, the higher contrast between the overlay and the cladding may lead to inaccuracies in the results obtained with the LP mode approximation (Del Villar et al. 2005 d). These inaccuracies occur when at specific ranges of overlay thickness values the modal field distribution is shifted towards the overlay (Del Villar et al. 2005 a). In that case the modal intensity is not negligible both in the overlay and the cladding, and the weak guidance condition is not satisfied due to the high index contrast between the 
two regions. As a result, a vectorial model is necessary (Del Villar et al. 2005 e). This model is an extension to a multilayer cylindrical waveguide of the three-layer-model proposed in (Erdogan 1997). The numerical method is explained in section 1 and it will be used henceforward.

The advantage of depositing materials of a high refractive index contrast with the cladding of the LPFG is that the sensitivity (defined as the ratio between the resonance wavelength shift and the parameter variation) to variations in the ambient refractive index or in the overlay thickness is highly increased (Del Villar et al. 2005 a). However, the drawback is that the sensitivity to variations in the overlay refractive index is reduced (Del Villar et al. 2005 d). That is the reason why in (Pilla et al. 2005 b), the deposition of a syndiotactic polystyrene (SPS), with low refractive index contrast with the cladding of an LPFG, is a good choice for the detection of wide resonance wavelength shifts when the refractive index of SPS is modified. In section 2 a design will be proposed that enhances the sensitivity of the LPFG in all cases (variation of the overlay thickness, overlay refractive index or ambient refractive index) without the necessity of depositing a material of high refractive index contrast with the cladding. After analyzing the evolution of the effective index distribution, the design should permit to increase the sensitivity of the device to almost two orders of magnitude. However, the vanishing of the attenuation band becomes clearer as the sensitivity is increased, which compels to concentrate in those cases where the attenuation bands are still visible. Finally, some concluding remarks are given in section 3. This is the first time to our knowledge that this phenomenon has been studied in the literature.

\section{Theory}

The three-layer model used in (Erdogan et al. 1997) for the analysis of LPFG structures surrounded by a medium of lower refractive index than the cladding of the LPFG 
can be extended to the analysis of LPFGs coated with one or several overlays (see Fig. 1). The application of the Transfer Matrix Method presented in (Guo et. al 2004), permits to calculate the propagation constants in the multilayer cylindrical waveguide. For the sake of simplicity, the structure simulated presents no azimuthal variation of the perturbed index profile after exposure to UV radiation. In this way, there are only interactions between the core mode $H E_{1 l}$, and $H E_{l j}$ and $E H_{l j}$ modes of the cladding (Erdogan et al. 1997). The notation used for the cladding modes will be henceforward $\mathrm{HE}_{1,2}$ for the first $\mathrm{HE}_{1, \mathrm{j}}$ cladding mode, $\mathrm{HE}_{1,4}$ for the second $\mathrm{HE}_{1, \mathrm{j}}$ cladding mode, and so on; $\mathrm{EH}_{1,3}$ for the first $\mathrm{EH}_{1, \mathrm{j}}$ cladding mode, $\mathrm{EH}_{1,5}$ for the second $\mathrm{EH}_{1, \mathrm{j}}$ cladding mode, and so on. After that, the next two steps are the same as those indicated in (Erdogan et al. 1997): calculation of the self- and cross-coupling coefficients of the modes, and calculation of the transmission spectrum after solving the coupled-mode differential equation:

$$
\left(\begin{array}{c}
\dot{F_{11}}(z) \\
\dot{F_{12}}(z) \\
\mathrm{M} \\
\dot{F_{1 N}}(z)
\end{array}\right)=\left(\begin{array}{cccc}
Q_{11} & V_{12,11} & \Lambda & V_{1 N, 11} \\
V_{11,12} & Q_{12} & \Lambda & V_{1 N, 12} \\
\mathrm{M} & \mathrm{M} & \mathrm{O} & \mathrm{M} \\
V_{11,1 N} & V_{12,1 N} & \Lambda & Q_{1 N}
\end{array}\right)\left(\begin{array}{c}
F_{11}(z) \\
F_{12}(z) \\
\mathrm{M} \\
F_{1 N}(z)
\end{array}\right)
$$

where $F_{l j}$ is the normalized amplitude of the $j$ mode, and the Differential Equation (DE) matrix elements are defined as:

$$
\begin{aligned}
& Q_{1 j}=-j \sigma(z) s_{0} \zeta_{1 j, 1 j} \\
& V_{1 j, 1 k}=-j \sigma(z) \frac{s_{1}}{2} \zeta_{1 j, 1 k} \exp \left[-j z\left(\beta_{1 j}-\beta_{1 k} \pm \frac{2 \pi}{\Lambda}\right)\right]
\end{aligned}
$$

Where $z$ is the longitudinal axis of the LPFG, $\sigma(z)$ is the apodization factor of the grating, $s_{0}$ and $s_{1}$ are the coefficients of the first two Fourier components of the grating 
function $S(z), \beta_{l j}$ is the propagation constant of the $j$ mode, $\varsigma_{l j, l k}$ is the coupling coefficient between the $j$ and the $k$ mode , and $\Lambda$ is the period of the grating.

There exist approximations that reduce the computation time of the algorithm. The first one is the calculation of the resonance wavelength with the Bragg condition:

$$
\beta_{11}(\lambda)-\beta_{1 j}(\lambda)=\frac{2 \pi}{\Lambda}
$$

where $\beta_{11}$ and $\beta_{l j}$ are the propagation constants of the core and the $j$ cladding modes respectively. This produces inexact results. However, if the modified first-order Bragg condition is applied, errors are lower than $0.1 \%$ (Anemogiannis et al. 2003):

$$
\beta_{11}(\lambda)+s_{0} \zeta_{11,11}(\lambda)-\left(\beta_{0 j}(\lambda)+s_{0} \zeta_{1 j, 1 j}(\lambda)\right)=\frac{2 \pi}{\Lambda}
$$

where $\varsigma_{11,11}$ and $\varsigma_{1 j, 1 j}$ are the self-coupling coefficients of the core and the $j$ cladding modes. However, because we are also interested in the analysis of the depth of the attenuation bands in the transmission spectrum we prefer the complete formulation (expression 1)

\section{LPFGs with two-overlay coating}

The experiments performed with LB, ESA and DC methods (Rees et al. 2002; Del Villar et al. 2005 b; Pilla et al. 2005 a) in LPFGs coated with a unique overlay, indicate that cladding modes with lower effective index than the one that is guided by the overlay will shift their effective index value towards the effective index of the immediate higher effective index mode. As more material is deposited, the effective index distribution of the bare LPFG is recovered (Rees et al. 2002; Del Villar et al. 2005 b). It must be considered that in the case of no azimuthal perturbation there is a correspondence between $\mathrm{LP}_{0, \mathrm{j}}$ modes and $\mathrm{HE}_{1, \mathrm{j}}$ modes. Consequently, each $\mathrm{HE}_{1, \mathrm{j}}$ mode will shift its effective index to that of the immediate lower 
order $\mathrm{HE}_{1, \mathrm{j}}$ mode of the LPFG without overlay. However, the transition of the effective index of each mode is actually performed in two steps. This fact is not considered with the LP mode approximation. There is an intermediate transition to the effective index of a $\mathrm{EH}_{1, \mathrm{j}}$ mode when an HE cladding mode is guided in the overlay, which is clearly appreciated if there is a high contrast between the cladding and the overlay refractive index (Del Villar et al. $2005 \mathrm{~d}$ ). The second transition occurs when an EH cladding mode is guided in the overlay.

In Fig. 1 an LPFG is coated with two overlays. Consequently, the cylindrical multilayer waveguide has five layers: core, cladding, low-refractive-index overlay, highrefractive-index-overlay and ambient. The term low-refractive-index means lower refractive index than the cladding and the term high-refractive-index means higher refractive index than the cladding. The sense for this design stems from the analogy between semiconductors theory and photonic crystals theory (Joannopoulos et al. 1995). The structure of Fig. 1 is not a photonic crystal, but the thin low-refractive-index overlay between the high-refractive-index overlay and the cladding, could be considered as a potential barrier in analogy to semiconductor tunnel diodes, where the barrier of potential must be very narrow.

In order to analyze the problem, the following parameters are selected for the LPFG: core diameter $5 \mu \mathrm{m}$, cladding diameter $125 \mu \mathrm{m}$, core refractive index 1.4573, cladding refractive index 1.45 , period of the grating $276 \mu \mathrm{m}$, and length of the grating $25 \mathrm{~mm}$. The modulation is considered sinusoidal with an amplitude of $2.7 \times 10^{-4}$, and the apodization factor is 1. The first overlay material is formed by electrostatic self-assembled layers of polyallylamine hydrochloride and Prussian blue $\left[\mathrm{PAH}+\right.$ Prussian $\left.\mathrm{Blue}^{+}\right]$and polyacrylic acid [PAA $\left.{ }^{-}\right]$with refractive index 1.37 (Del Villar et al. 2005 c). The second overlay material is formed by electrostatic self-assembled layers of polyallylamine hydrochloride $\left[\mathrm{PAH}^{+}\right]$and 
polyacrylic acid $\left[\mathrm{PAA}^{-}\right]$with refractive index 1.55 . Both refractive index values have been considered constant in the range of wavelengths analyzed.

In Fig. 2 the effective index of the first six cladding modes is represented as a function of the high-refractive-index overlay for two different cases: a structure with a unique highrefractive-index overlay (first overlay of $0 \mathrm{~nm}$ ), and a structure with a first low-refractive index overlay of $450 \mathrm{~nm}$. The two-step transition starts for higher second overlay thickness values and it is more abrupt when a low-refractive index overlay is set between the cladding and the high-refractive-index overlay. The low-refractive-index layer between the highrefractive-index overlay and the cladding of the LPFG actuates as a barrier that retards the guidance of the first cladding mode in the overlay. Finally, a cladding mode tunnels through the low-refractive-index overlay and becomes guided in the high-refractive-index overlay. Once the mode is guided in the overlay, its effective index trends to the refractive index of the second overlay faster than in the case where no low-refractive-index overlay is present. This causes a more abrupt modal redistribution, which permits to observe the two-step transition without the necessity of depositing a material with a high refractive index contrast with the LPFG cladding. If some first overlay thickness values are analyzed, it seems that the maximum derivative of the effective index of the modes with respect to the second overlay thickness grows exponentially without a limit. As an example, in Fig. 3 it is analyzed the $\mathrm{HE}_{1,6}$ mode. However, a careful analysis of the same $\mathrm{HE}_{1,6}$ mode performed in Fig. 4 proves that there is a limit value. This value increases the maximum derivative of the effective index with respect to the second overlay thickness by a factor of 78 . This means nearly two orders of magnitude, which could be also interesting for sensing purposes in other structures such as slab waveguides (Stewart et al. 1994). We have also checked in our simulations that the results for the rest of modes are qualitatively the same. 
The variation of the effective indices of the cladding modes has an important effect in the attenuation bands of the transmission spectrum of the LPFG because there is a close relation between the wavelengths of these bands and the modified Bragg condition (Anemogiannis et al. 2003).

The consequence of the effective index variation is a displacement in all the attenuation bands. The attenuation band corresponding with the $\mathrm{HE}_{1,16}$ (respectively $\mathrm{LP}_{0,9}$ mode) shifts the wavelength to that of the $\mathrm{HE}_{1,14}$ (respectively $\mathrm{LP}_{0,8}$ mode); the same is true for the $\mathrm{HE}_{1,14}$ (respectively $\mathrm{LP}_{0,8}$ mode) that shifts the wavelength to the attenuation band of the $\mathrm{HE}_{1,12}$ (respectively $\mathrm{LP}_{0,7}$ mode), and so forth.

Focus will be centered now on the $\mathrm{HE}_{1,16}$ mode (fifteen cladding mode) resonance, because it permits to obtain a wide wavelength shift in the transmission spectrum. In Fig. 5a the $\mathrm{HE}_{1,16}$ mode resonance wavelength is represented as a function of the second overlay thickness for four different first overlay thicknesses: 0 (no barrier), 150, 300 and $450 \mathrm{~nm}$. The two-step transition observed in Fig. 2 occurs also in Fig. 5 due to the relation existent between the resonance wavelength and the effective index of cladding modes (see expression 4). Due to this two-step transition, two clear optimum overlay thickness (OOT) values (one for each transition) are obtained with an enhanced sensitivity to variations in the second overlay thickness. Unfortunately, in Fig. 5b it is shown that the vanishing of the attenuation band is more important if a barrier overlay is deposited between the high-refractive-index overlay and the cladding. This factor limits the sensitivity improvement to those low-refractive-index overlay thickness values that guarantee that the attenuation band is still visible. Another crucial factor is the tolerances of the overlay parameters which in the cases of an enhanced sensitivity (wider resonance wavelength shift as a function of the parameter variation) become more critical for defining an optimum sensor design. 
On the other hand, the question remains open whether the tunneling of the cladding mode improves the sensitivity of the device to variations in the second overlay refractive index. In Fig. 6 we compare the resonance wavelength shift as a function of the variation in the overlay refractive index for two cases: a structure with no barrier overlay and another one with a barrier of thickness $450 \mathrm{~nm}$ and refractive index 1.37 . The main OOT in both cases is 427 and $652 \mathrm{~nm}$ respectively. At the main OOT, the overall wavelength shift is improved from $30.6 \mathrm{~nm}$ in the case where no barrier is present to $104.8 \mathrm{~nm}$ in the case where there is a barrier overlay.

Once it has been proved that the inclusion of a low-refractive-index overlay between the high-refractive-index overlay and the cladding of the LPFG improves the sensitivity to variations in the refractive index and the thickness of the high-refractive-index of the overlay, the last question is whether the sensitivity to ambient refractive index variations could also be improved. To this purpose the two designs of Fig. 6 will be analyzed in Fig. 7. Five ambient refractive indices are analyzed: 1, 1.005, 1.01, 1.015 and 1.02. The overall wavelength shift originated is $3.7 \mathrm{~nm}$ for the case where there is no barrier overlay and of $30.6 \mathrm{~nm}$ in the other case. This constitutes an improvement factor of 8.27.

Finally it is important to mention that other sensor fields where LPFGs are used, such as the detection of temperature and strain variations could also benefit from the application of low-refractive-index and high-refractive-index overlays. The explanation is that the method for the detection consists of the variation in the effective index of the cladding modes. This variation could be enhanced by the deposition of adequately parameterized overlays on the LPFG cladding (James and Tatam 2001; Bathia 1999). 


\section{Conclusion}

In summary, the inclusion of a thin low-refractive-index overlay between a thin highrefractive-index overlay and the cladding of an LPFG acts as a barrier preventing the guidance of a cladding mode in the overlay. Finally, the mode tunnels through the lowrefractive-index overlay and is guided in the high-refractive index overlay. This causes a more abrupt modal redistribution, which resembles the modal redistribution of an LPFG with deposition of an overlay of a very high refractive index. The consequence is a sensitivity enhancement of LPFGs with overlay deposition in all cases: variation of the overlay refractive index, of the overlay thickness and of the ambient refractive index. The limit in the sensitivity improvement is marked by the vanishing of the attenuation bands, but in other structures without this limitation, the sensitivity improvement could reach almost two orders of magnitude. This design solves the problem of LPFGs coated with a simple high-refractive index overlay, where the sensitivity to overlay refractive index variations is reduced, and avoids the necessity of materials of higher refractive indices that those typical of polymers.

The theory explained in this work could be extrapolated to other sensor fields where LPFGs are used, such as temperature and strain detection. In these other cases the overall wavelength shift could also be enhanced with the deposition of low-refractive and high-refractive index overlays on the cladding.

\section{Acknowledgements}

This work was supported by Spanish Ministerio de Ciencia y Tecnologia and FEDER Research Grants CICYT-TIC 2003-00909, Gobierno de Navarra and FPU MECD Grant. 


\section{References}

Anemogiannis, E., Glytsis, E.N. and Gaylord, T.K. J. Lightwave Technol. 21 218, 2003.

Bhatia, V. Opt. Express 4 457, 1999.

Del Villar, I., Matias, I.R., Arregui, F.J. and Lalanne, P. Opt. Express 13 56, 2005.

Del Villar, I., Achaerandio, M., Matias, I. R. and Arregui, F. J., Opt. Lett. 30 720, 2005.

Del Villar, I., Matias, I.R., Arregui, F.J. and Claus, R.O. IEEE Trans. Nanotehcnol. 4 187, 2005.

Del Villar, I., Matias, I.R. and Arregui, F.J. IEEE Photon. Technol. Lett. 17 1893, 2005.

Del Villar, I., Matias, I.R. and Arregui, F.J. J. Opt. Soc. Am A. In press.

Erdogan T. J. Opt. Soc. Am. A 14 1760, 1997.

Guo, S., Albin, S. and Rogowski, R.S. Opt. Express 12 198, 2004.

James, S.W. and Tatam, R.P. Meas. Sci Technol. 14 R49, 2003.

Joannopoulos, J.D., Meade, R.D. and Winn, J.N. Photonic crystals: molding the flow of light, Chap. 1, Princeton

U. Press., Princeton, N. J., 1995.

Pilla, P., Contessa, L., Iadicicco, A., Campopiano, S., Cutolo, A., Giordano, M. and Cusano, A. Proc. of IEEE/LEOS Workshop on Fibers and Optical Passive Components., 370, 2005.

Pilla, P., Iadicicco, A., Contessa, L., Campopiano, S., Cutolo, A., Giordano, M., Guerra, G. and Cusano, A., IEEE Photon. Technol. Lett. 17 1713, 2005.

Rees, N.D., James, S.W., Tatam, R.P. and Ashwell, G.J. Opt. Lett. 27 686, 2002.

Stewart, G., and Culshaw, B. Opt. Quant. Elec. 26 S249, (1994). 


\section{Figure captions}

\section{Figure 1.}

Longitudinal and transversal section of LPFG structure with two-overlay coating, and index profile of the transversal section.

\section{Figure 2.}

Effective index as a function of the overlay thickness of first six cladding modes for: a) an LPFG with one overlay of refractive index 1.55. b) an LPFG with a first overlay of $450 \mathrm{~nm}$ and refractive index 1.37, and a second overlay with refractive index 1.55.

\section{Figure 3.}

Derivative of the effective index as a function of the overlay thickness for the $\mathrm{HE}_{1,6}$ mode in: a) an LPFG with one overlay of refractive index 1.55 . b) an LPFG with a first overlay of 150 $\mathrm{nm}$ and refractive index 1.37 and a second overlay of refractive index 1.55. c) an LPFG with a first overlay of $300 \mathrm{~nm}$ and refractive index 1.37 and a second overlay of refractive index 1.55. d) an LPFG with a first overlay of $450 \mathrm{~nm}$ and refractive index 1.37 and a second overlay of refractive index 1.55 .

\section{Figure 4.}

For an LPFG coated with one overlay of refractive index 1.37 and a second one of refractive index 1.55: a) maximum derivative of the effective index of the $\mathrm{HE}_{1,6}$ mode with respect to the second overlay as a function of the first overlay thickness b) second overlay thickness to 
obtain a maximum derivative of the effective index of the $\mathrm{HE}_{1,6}$ mode with respect to the second overlay thickness as a function of the first overlay thickness.

\section{Figure 5.}

An LPFG with two overlays: a) $\mathrm{HE}_{1,16}$ resonance wavelength and $\mathrm{b}$ ) $\mathrm{HE}_{1,16}$ minimum transmission as a function of the second overlay thickness for four different first overlay thickness values. First and second overlay refractive indices: 1.37 and 1.55 respectively. Ambient refractive index: 1 .

\section{Figure 6.}

a) An LPFG with one overlay: $\mathrm{HE}_{1,16}$ resonance wavelength as a function of the thickness of the unique overlay Three overlay refractive indices are analyzed: $1.545,1.55$ and 1.555. b) An LPFG with two overlays: HE, 1,16 resonance wavelength as a function of the second overlay thickness. Three overlay refractive indices are analyzed: $1.545,1.55$ and 1.555 . The thickness and the refractive index of the first overlay are $450 \mathrm{~nm}$ and 1.37 respectively. Ambient refractive index 1 .

\section{Figure 7.}

$\mathrm{HE}_{1,16}$ resonance wavelength as a function of the ambient refractive index for a) an LPFG with one overlay of $427 \mathrm{~nm}$ and refractive index 1.55 . b) an LPFG with a first overlay of 450 $\mathrm{nm}$ and refractive index 1.37 and a second overlay of $652 \mathrm{~nm}$ and refractive index 1.55. 


\section{Figures}

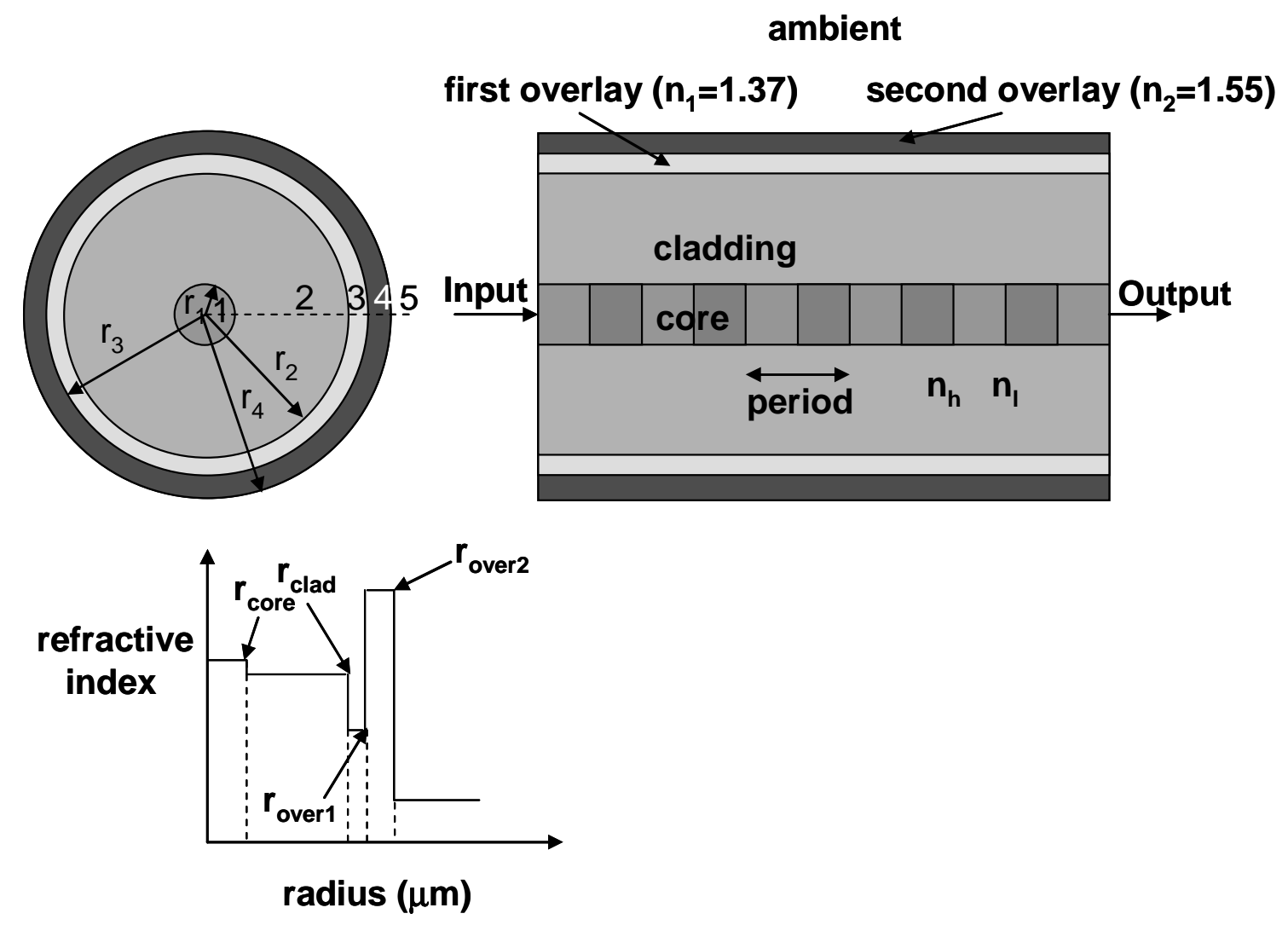

Figure 1. 


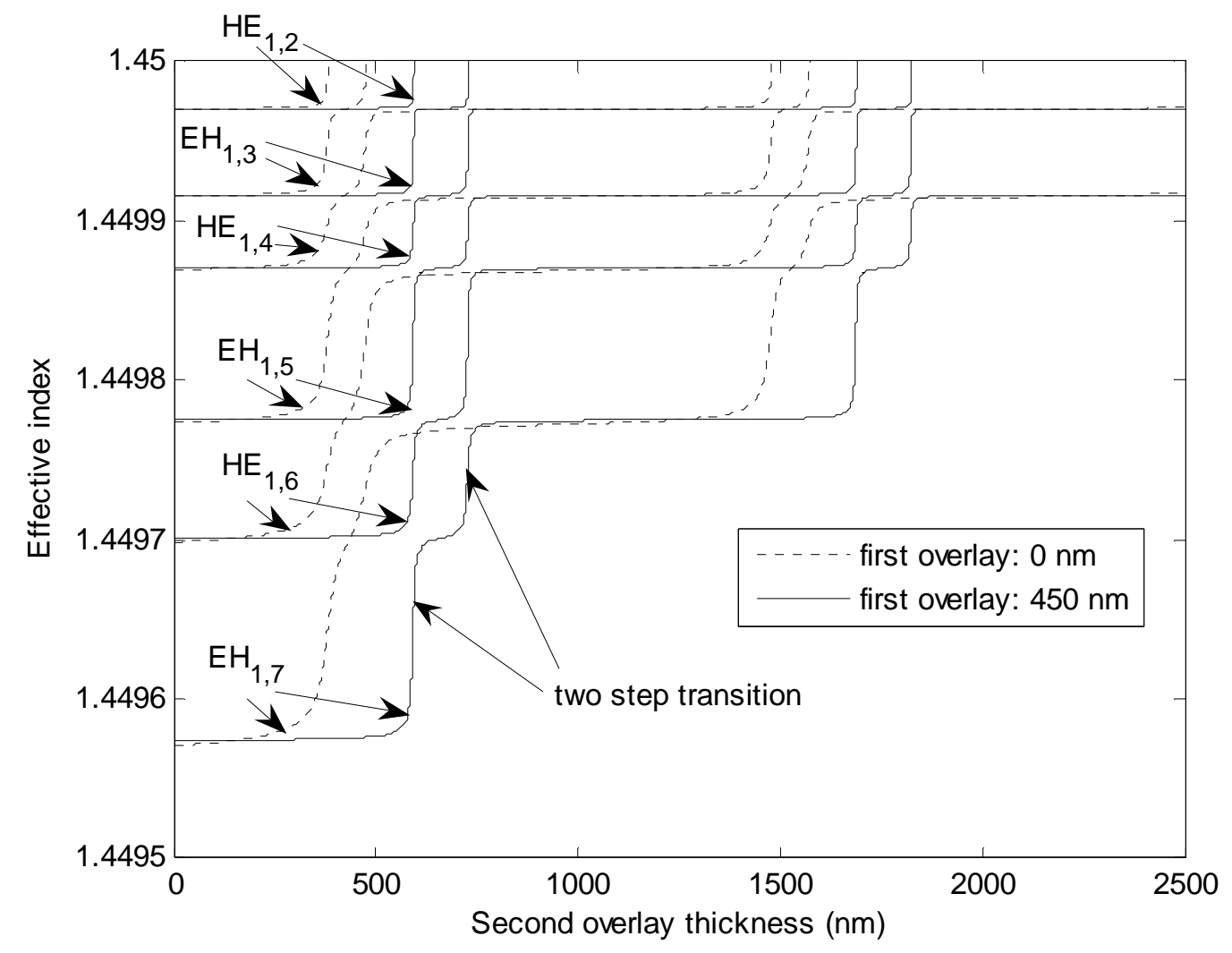

Figure 2 


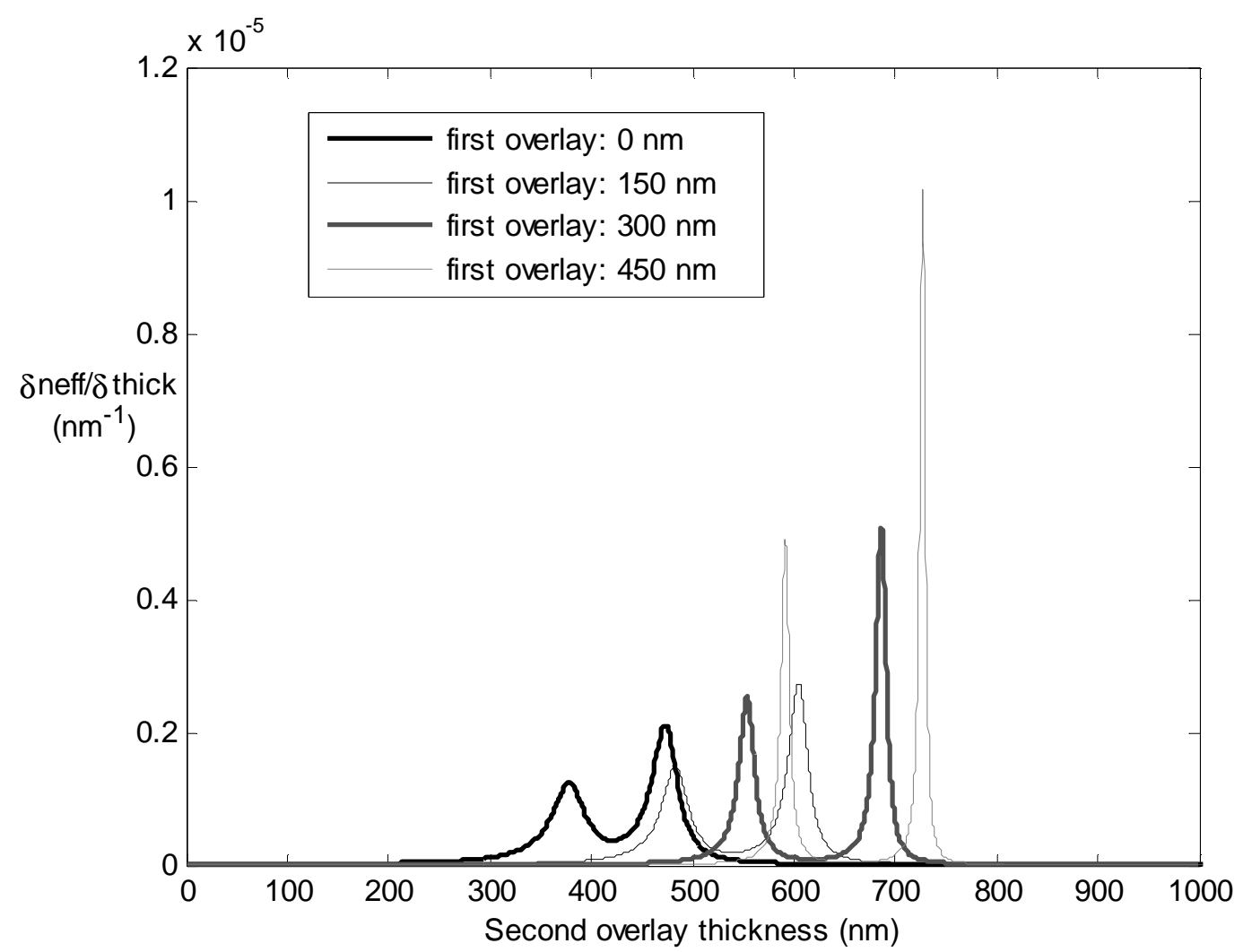

Figure 3 


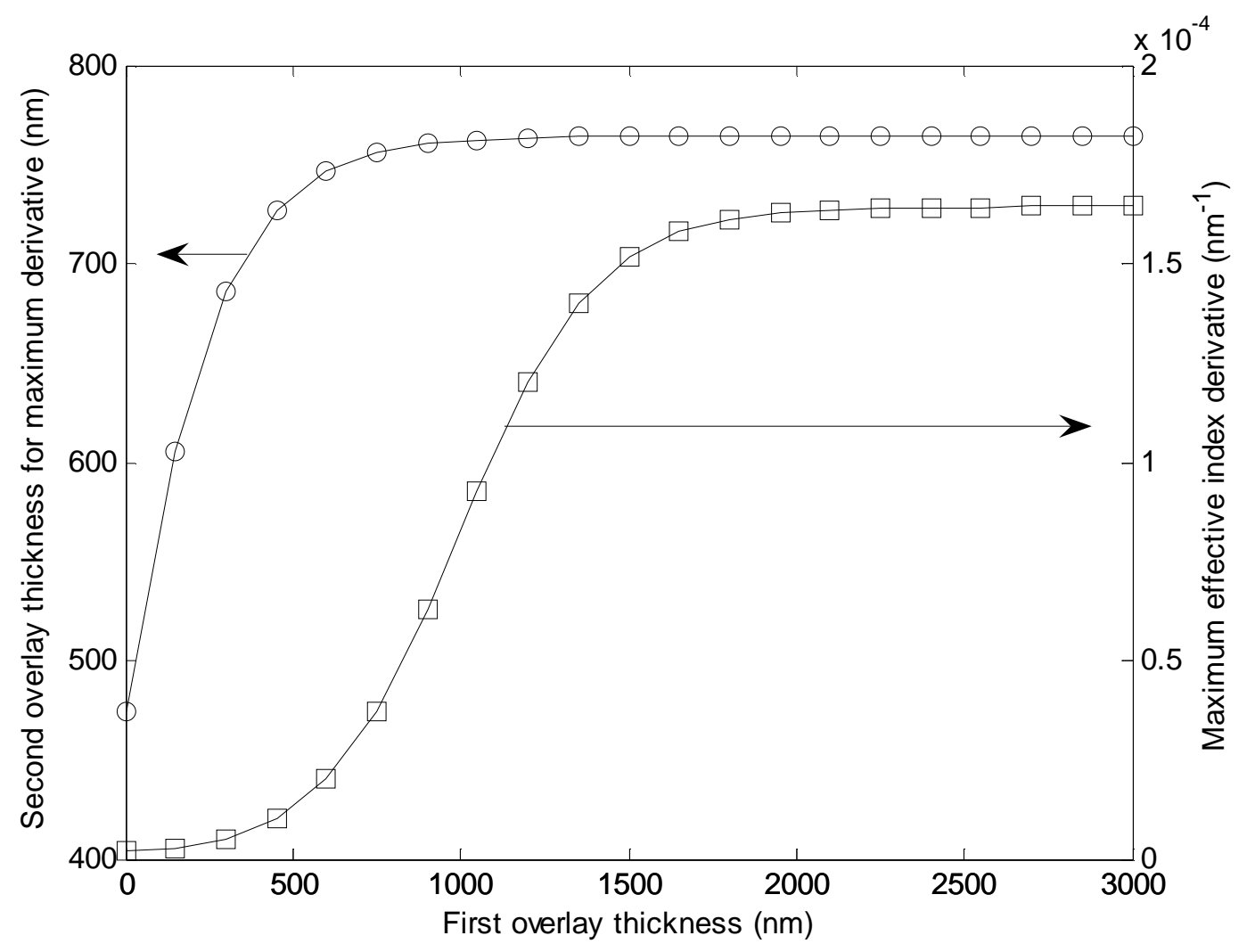

Figure 4 

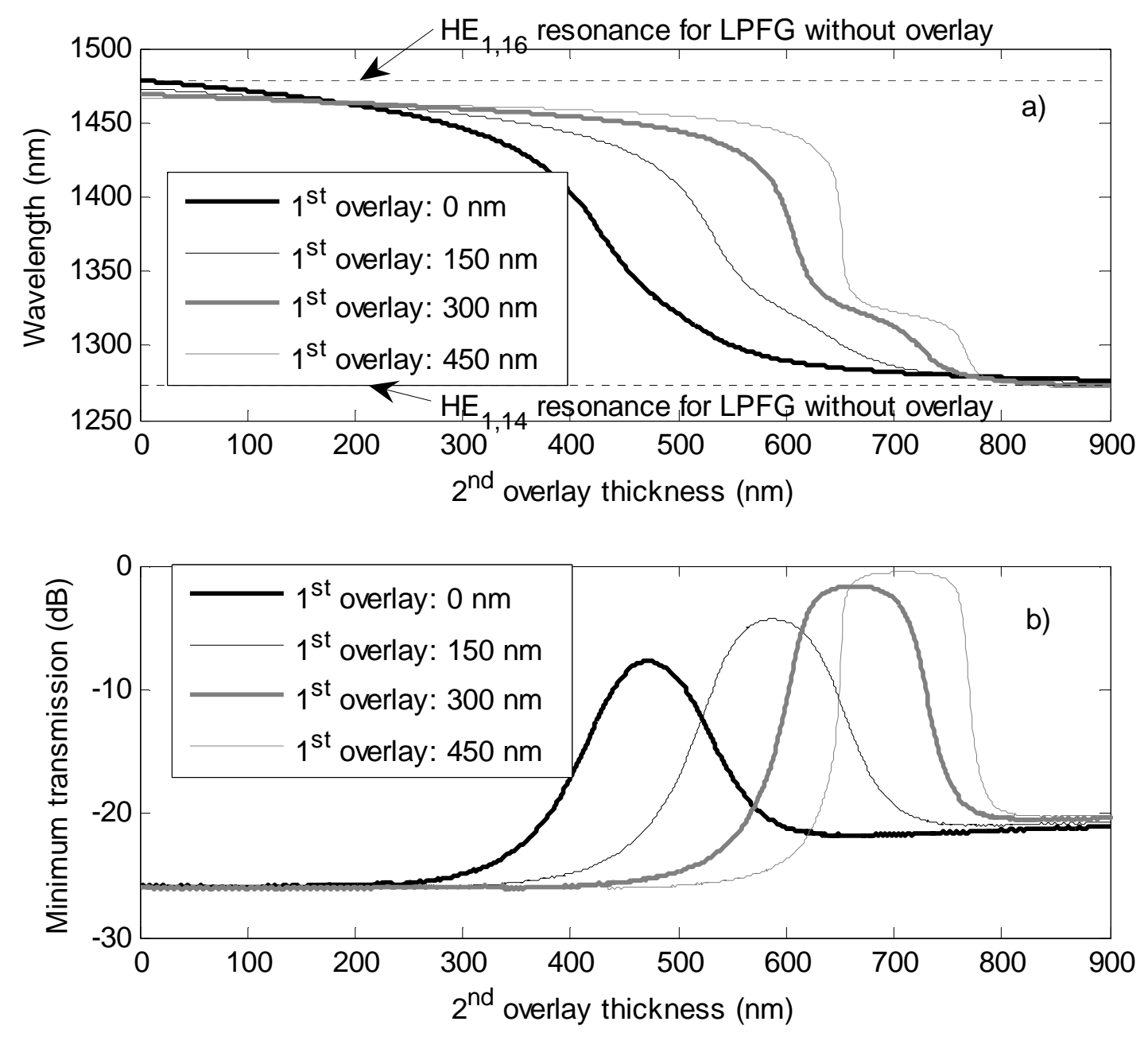

Figure 5 

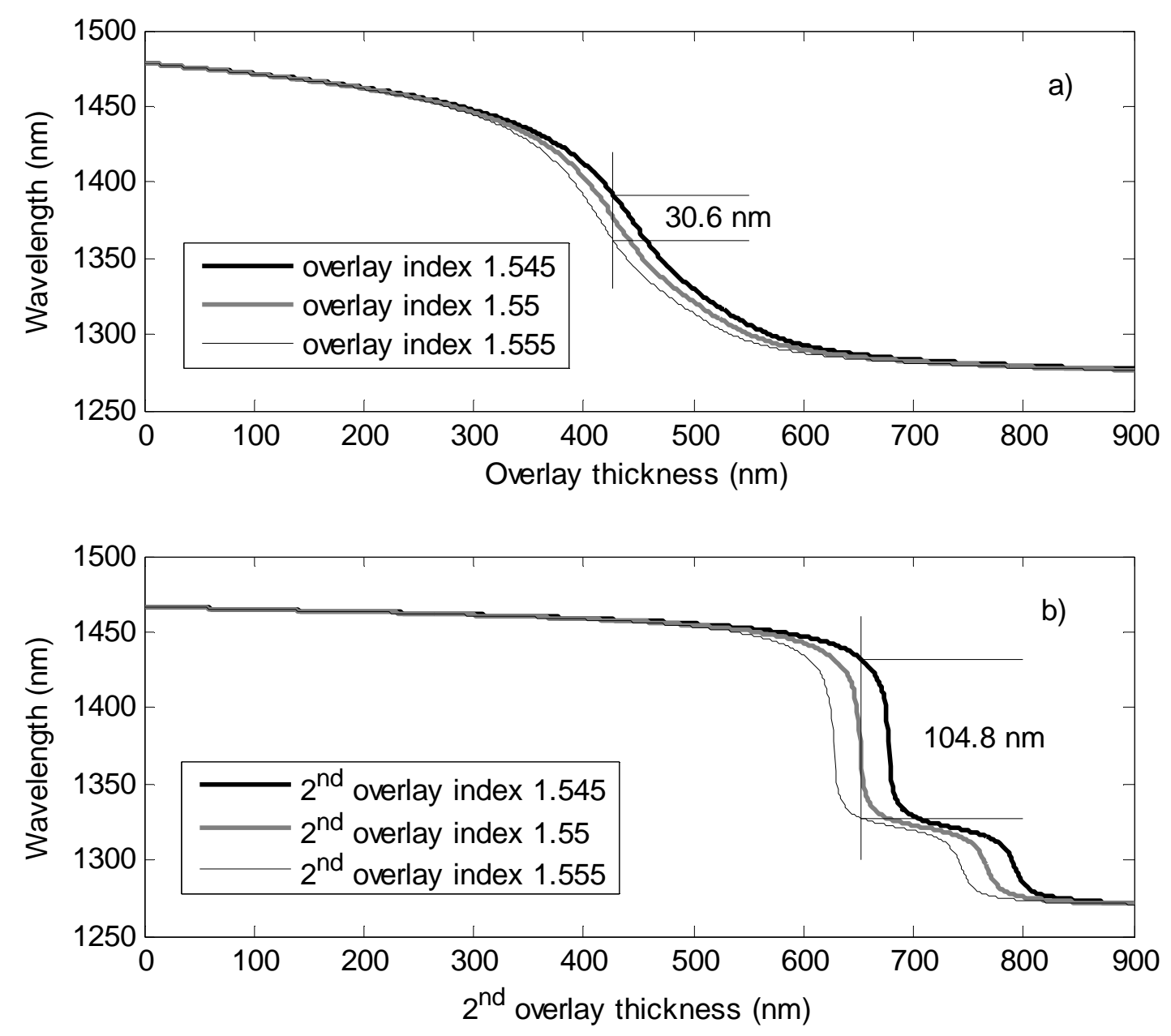

Figure 6 


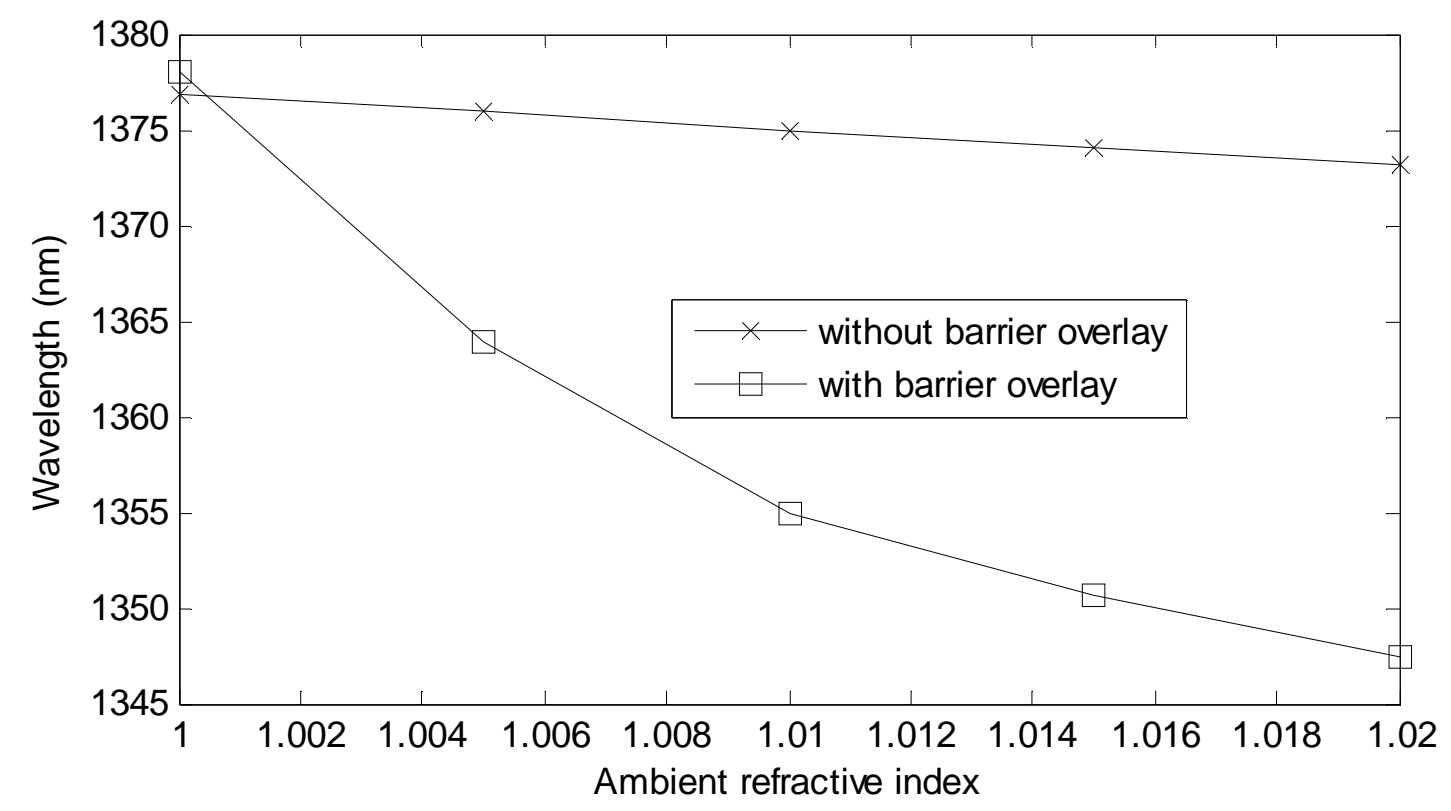

Figure 7 